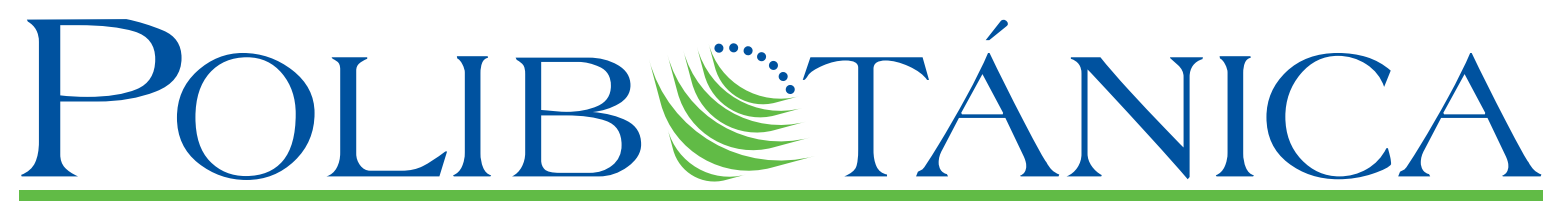

\title{
ISSN 1405-2768
}

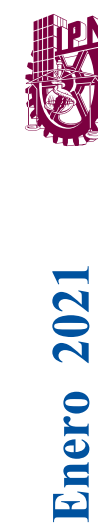

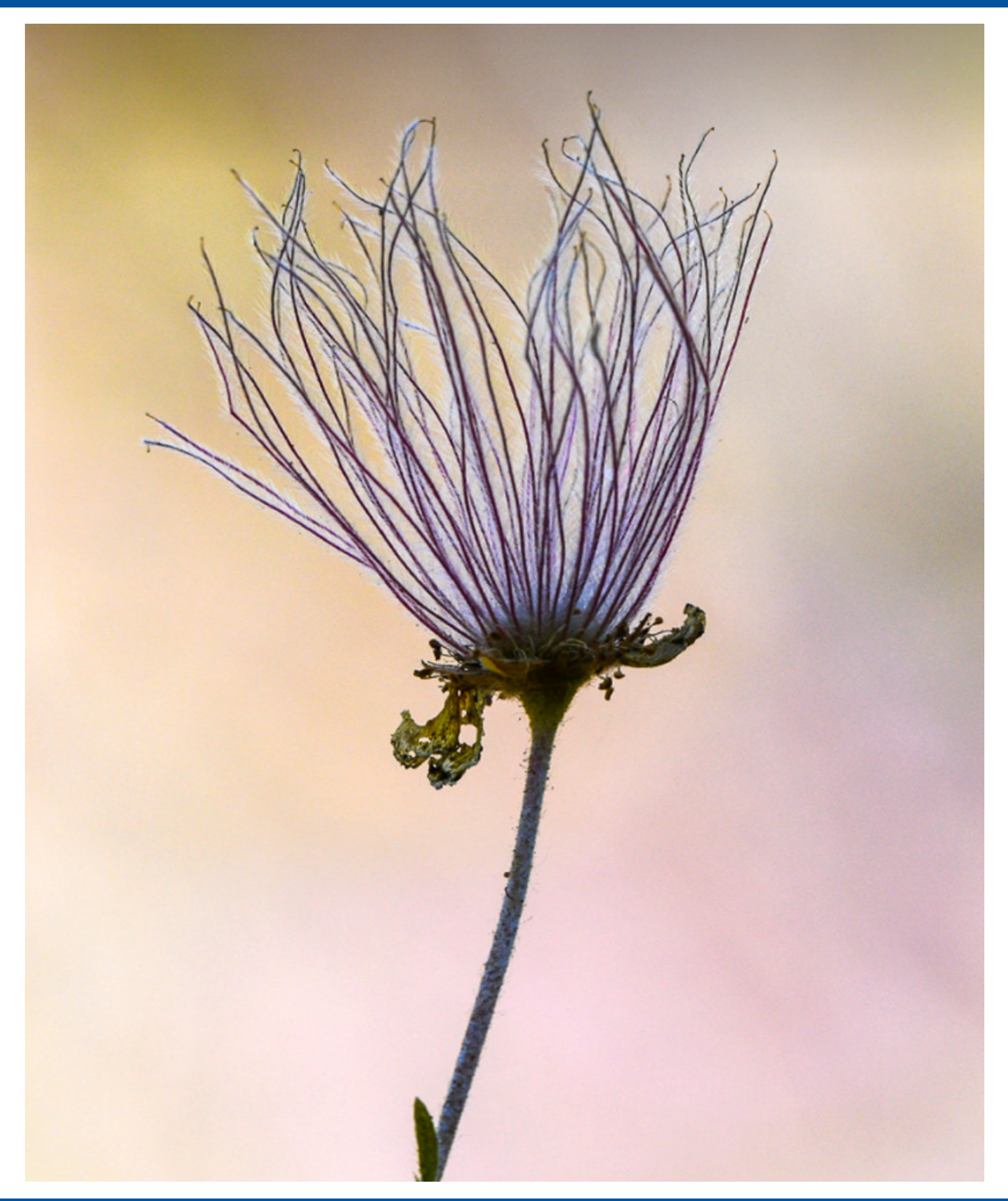

Núm. 51 
PÁG.

CONTENIDO

1 La familia Rosaceae en México.

The Rosaceae family in Mexico.

Rzedowski, J.

17 Orquídeas de los municipios de Santo Domingo Yanhuitlán y San Pedro y San Pablo Teposcolula, Oaxaca. Orchids from the municipalities of Santo Domingo Yanhuitlan and San Pedro y San Pablo Teposcolula, Oaxaca. Ibarra-Contreras, C.A. | R. Solano | L. Paz-Cruz |C. Pérez-Domínguez | L. Lagunez-Rivera

43 Caracterización de la variación morfológica de Cyanotetras (Cyanobacteria) en el Lago de Catemaco Veracruz, México. Characterization of morphological variation of Cyanotetras (Cyanobacteria) in Catemaco Lake, Veracruz, Mexico. Muciño-Márquez, R.E. | R.O. Echenique | I. Gárate-Lizárraga| M.G. Figueroa-Torres |A. Esquivel-Herrera

55 Estructura vertical de un bosque de galería en un gradiente altitudinal en el noroeste de México. Vertical structure of a gallery forest on an altitude gradient in northwestern of Mexico. Holguín-Estrada, V.A. | Alanís-Rodríguez E. | Aguirre-Calderón O.A. | J.I. Yerena-Yamallel | M.Á. Pequeño-Ledezma

73 Diversidad arbórea nativa: base para el diseño de sistemas agroforestales en una comunidad maya en la Península de Yucatán, México.

Native tree diversity: basis for the design of agroforestry systems in a mayan community in the Yucatan Peninsula, Mexico. Dzib-Castillo, B. B. | H. Van der Wal |V. Cervantes-Gutiérrez | W. Cetzal-Ix | C. I. Chanatásig-Vaca|F. Casanova-Lugo

91 Estructura y diversidad arbórea de un bosque de galería urbano en el Río Camachito, noreste de México.

Tree structure and diversity of an urban gallery forest in the Camachito River, northeast Mexico.

Canizales-Velázquez, P.A. | E. Alanís-Rodríguez | S. A. García-García| V. A. Holguín-Estrada|A. Collantes-Chávez-Costa

107 Estructura y diversidad del matorral xerófilo en el noreste de México. Structure and diversity of the xerophilous scrub in northeast Mexico.

Reyna-González, A. M. | P.S. Soto-Borrego | E. Alanís-Rodríguez | V. M. Molina-Guerra | A. Collantes-Chávez-Costa

123 Fenología floral de nueve variedades de nochebuena (Euphorbia pulcherrima Willd. ex Klotzsch). Floral phenology of nine poinsettia varieties (Euphorbia pulcherrima Willd. ex Klotzsch). Rodríguez-Rojas, T. de J. | M. Andrade-Rodríguez | A. Castillo-Gutiérrez | O.G. Villegas-Torres

141 Micropropagación del lirio amazónico (Eucharis grandiflora Planch. \& Linden) mediante organogénesis directa. Micropropagation of amazon lily (Eucharis grandiflora Planch. \& Linden) through direct organogenesis.

Guerrero-Valencia, F.A. | J.L. Rodríguez-de Ta O | M. de J. Juárez-Hernández | J. Ayala-Arreola |G. Ramírez-González

155 Caracterización micrográfica de tres frutos tropicales, Musa paradisii L., Persea americana Mill. y Physalis peruviana L. Importancia en el control de calidad botánico de alimentos derivados.

Micrographic characterization of three tropical fruits, Musa paradisii L., Persea americana Mill. and Physalis peruviana $L$. Significance in the definition of botanical quality of derived foods.

Gimenez, L. A. S. | M. A. Rivas | N. D. Vignale |A. A. Gurni

171 Semillas de rabanitos (Raphanus sativus L): observaciones de su morfología bajo microscopía electrónica, germinación y utilidad para estudios de fitotoxicidad.

Seeds of radishes (Raphanus sativus L): observations of its morphology under electron microscopy, germination and usefulness for phytotoxicity studies.

Taladrid, I. J. M.B. Espinosa

185 Diversidad genética de la chaya (Cnidoscolus aconitifolius (Mill.) I. M. Johnst. ssp. aconitifolius) en Yucatán, México, su posible centro de domesticación.

Genetic diversity of chaya (Cnidoscolus aconitifolius (Mill.) I. M. Johnst. ssp. aconitifolius) in Yucatan, Mexico, its putative domestication center.

Chin-Chan, T. | M.M. Ortiz-García | P.J. Ruiz-Gil | J. Martínez-Castillo

203 Caracterización química de los volátiles de Tagetes nelsonii.

Chemical characterization of the volatiles of Tagetes nelsonii.

Cruz Flores, O.| M. Espinoza Ruiz | A. Santiesteban Hernández | L. Cruz-López

213 Extractos vegetales para el control de Colletotrichum gloeosporioides in vitro, en periodo de floración y poscosecha del fruto de Carica papaya.

Plant extracts for control of Colletotrichum gloeosporioides in vitro and flowering period and postharvest stage on Carica papaya. García-Mateos, M.R. | M. Acosta-Ramos | E. Rodríguez-Pérez | J. Vásquez-Sanchez | L. Hernández-Ramos

229 Importancia relativa de frutos y verduras comercializadas en el Mercado de Izúcar de Matamoros, Puebla, México. Relative importance of fruits and vegetables commercialized in the Izúcar de Matamoros Market, Puebla, Mexico. Martínez Moreno, D. | J. Reyes Matamoros | A.L. López Pérez | F. Basurto Peña 


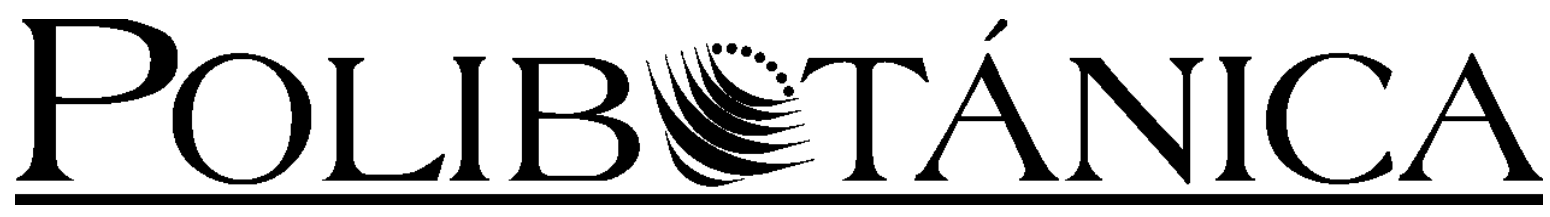

Núm. 51

ISSN electrónico: 2395-9525

Enero 2021

\section{Portada}

Fallugia paradoxa (D. Don) Endl. ex Torr. Rosaceae. "Penacho de Apache"

Arbustos de hasta $2 \mathrm{~m}$ de altura. Hojas de 6-8 $\mathrm{mm}$ de largo, en fascículos, usualmente caducas. Flores blancas y vistosas, generalmente unisexuales de $3 \mathrm{~cm}$ de diámetro. Aquenios obovoides-fusiformes, cada uno con un estilo que se modifica en una pluma rosada o violácea, retorcida y vellosa, de hasta $3 \mathrm{~cm}$ de largo, la "pluma" ayuda a dispersar las semillas por el viento. Prospera en matorral xerófilo. Se usa en la cestería para construir cunas, escobas y flechas, se considera valiosa para el control de la erosión en las zonas desérticas. Distribución: Noroeste de México y Sur de Estados Unidos.

Shrubs up to $2 \mathrm{~m}$ tall. Leaves 6-8 $\mathrm{mm}$ long, in fascicles, usually deciduous. Flowers white and showy, generally unisexual, 3 $\mathrm{cm}$ in diameter. Achenes obovoid-fusiform, each with a style that is modified into a pink or purplish, twisted and hairy feather, up to $3 \mathrm{~cm}$ long, the "feather" helps to disperse the seeds by the wind. Prosper in xerophilous scrub. It is used in basketry to build cradles, brooms and arrows, it is considered valuable for erosion control in desert areas. Distribution Northwest of Mexico and South of the USA.

por/by Rafael Fernández Nava 

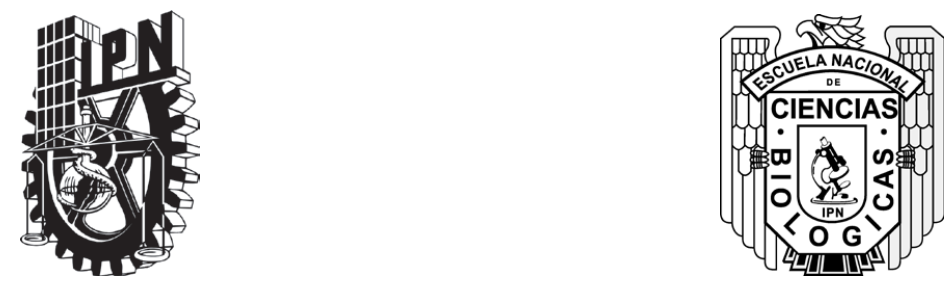

\title{
Instituto PolitéCNICO NACIONAL
}

Director General: Dr. Arturo Reyes Sandoval

Secretaria General: M.en D.D.C. María Guadalupe Vargas Jacobo

Secretario Académico: Dr. Jorge Toro González

Secretario de Extensión e Integración Social: Dr. Luis Alfonso Villa Vargas

Secretario de Investigación y Posgrado: Dr. Juan Silvestre Aranda Barradas

Secretario de Servicios Educativos: Dra. Ana Lilia Coria Páez

Secretario de Administración: C.P. Jorge Quintana Reyna

Director de Educación Superior: Ing. Juan Manuel Velázquez Peto

\section{ESCUEla NACIONAL DE Ciencias BiolóGiCAS}

\author{
Director: \\ Dr. Gonzalo Trujillo Chávez \\ Subdirectora Académica: \\ M. en C. Martha Patricia Cervantes Cervantes \\ Subdirector Administrativo: Ing. Raúl Chávez Alvircio \\ Jefe de la Sección de Estudios de Posgrado e Investigación: \\ Dr. Gerardo Aparicio Ozores
}

POLIBOTÁNICA, Año 26, No. 51, enero-junio 2021, es una publicación semestral editada por el Instituto Politécnico Nacional, a través de la Escuela Nacional de Ciencias Biológicas. Unidad Profesional Lázaro Cárdenas, Prolongación de Carpio y Plan de Ayala s/n, Col. Santo Tomas C.P. 11340 Delegación Miguel Hidalgo México, D.F. Teléfono 57296000 ext. 62331.

http://www.herbario.encb.ipn.mx/, Editor responsable: Rafael Fernández Nava. Reserva de Derechos al Uso Exclusivo del Título No. 04-2015-011309001300-203. ISSN impreso: 1405-2768, ISSN digital: 2395-9525, ambos otorgados por el Instituto Nacional del Derecho de Autor. Responsable de la última actualización de este número, Unidad de informática de la ENCB del IPN, Rafael Fernández Nava, Unidad Profesional Lázaro Cárdenas, Prolongación de Carpio y Plan de Ayala s/n, Col. Santo Tomas C.P. 11340 Delegación Miguel Hidalgo México, D.F.

Las opiniones expresadas por los autores no necesariamente reflejan la postura del editor de la publicación.

Queda estrictamente prohibida la reproducción total o parcial de los contenidos e imágenes de la publicación sin previa autorización del Instituto Politécnico Nacional. 


\section{REVISTA BOTÁNICA INTERNACIONAL DEL INSTITUTO POLITÉCNICO NACIONAL}

EDITOR EN JEFE

Rafael Fernández Nava

EDITORA ASOCIADA

María de la Luz Arreguín Sánchez

Christiane Anderson

University of Michigan

Ann Arbor. Michigan, US

Heike Vibrans

Colegio de Postgraduados

Estado de México, México

Graciela Calderón de Rzedowski

Instituto de Ecología del Bajío

Páztcuaro, Mich., México

Theodore S. Cochrane

University of Wisconsin

Madison, Wisconsin, US

Hugo Cota Sánchez

University of Saskatchewan

Saskatoon, Saskatchewan, Canada

Fernando Chiang Cabrera

Universidad Nacional Autónoma de México

Ciudad de México, México

Thomas F. Daniel

California Academy of Sciences

San Francisco, California, US

Francisco de Asis Dos Santos

Universidad Estadual de Feira de Santana

Feira de Santana, Brasil

Carlos Fabián Vargas Mendoza

Instituto Politécnico Nacional

Ciudad de México, México

José Luis Godínez Ortega

Universidad Nacional Autónoma de México

Ciudad de México, México

José Manuel Rico Ordaz

Universidad de Oviedo

Oviedo, España
Edith V. Gómez Sosa

Instituto de Botánica Darwinion

Buenos Aires, Argentina

Jorge Llorente Bousquets

Universidad Nacional Autónoma de México

Ciudad de México, México

Delia Fernández González

Universidad de León

León, España

Jerzy Rzedowski Rotter

Instituto de Ecología del Bajío

Pátzcuaro, Mich., México

Luis Gerardo Zepeda Vallejo

Instituto Politécnico Nacional

Ciudad de México, México

Claude Sastre

Muséum National d'Histoire Naturelle

París, Francia

Mauricio Velayos Rodríguez

Real Jardín Botánico

Madrid, España

Noemí Waksman de Torres

Universidad Autónoma de Nuevo León

Monterrey, NL, México

Julieta Carranza Velázquez

Universidad de Costa Rica

San Pedro, Costa Rica

Tom Wendt

University of Texas

Austin, Texas, US

\section{DISEÑO Y FORMACIÓN ELECTRÓNICA}

Luz Elena Tejeda Hernández

\section{OPEN JOURNAL SYSTEM Y TECNOLOGÍAS DE LA INFORMACIÓN}


Toda correspondencia relacionada con la revista deberá ser dirigida a:

\section{Dr. Rafael Fernández Nava}

Editor en Jefe de

\section{POLIBUTÁNÁNACA}

Departamento de Botánica

Escuela Nacional de Ciencias Biológicas, Instituto Politécnico Nacional

Apdo. Postal 17-564, CP 11410, Ciudad de México

Correo electrónico:

polibotanica@gmail.com

rfernan@ipn.mx

Dirección Web

http://www.polibotanica.mx

POLIBOTÁNICA es una revista indexada en:

CONACYT, índice de Revistas Mexicanas de Investigación Científica y Tecnológica del Consejo Nacional de Ciencia y Tecnología.

SciELO - Scientific Electronic Library Online.

Google Académico - Google Scholar.

DOAJ, Directorio de Revistas de Acceso Público.

Dialnet portal de difusión de la producción científica hispana.

REDIB Red Iberoamericana de innovacion y conocimiento cientifico.

LATINDEX, Sistema regional de información en línea para revistas científicas de América Latina, el Caribe, España y Portugal.

PERIODICA, (Indice de Revistas Latinoamericanas en Ciencias). 


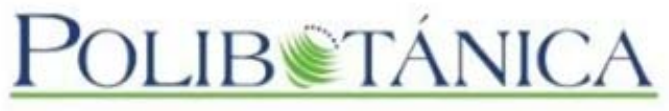

\section{Polibotánica}

ISSN electrónico: 2395-9525

polibotanica@gmail.com

Instituto Politécnico Nacional

México

http://www.polibotanica.mx

\section{DIVERSIDAD ARBÓREA NATIVA: BASE PARA EL DISEÑO DE SISTEMAS AGROFORESTALES EN UNA COMUNIDAD MAYA EN LA PENÍNSULA DE YUCATÁN, MÉXICO}

\section{NATIVE TREE DIVERSITY: BASIS FOR THE DESIGN OF AGROFORESTRY SYSTEMS IN A MAYAN COMMUNITY IN THE YUCATAN PENINSULA, MEXICO}

B. B. Dzib-Castillo, H. Van der Wal, V. Cervantes-Gutiérrez, W. Cetzal-Ix, C. I. Chanatásig-Vaca y F. Casanova-Lugo.

DIVERSIDAD ARBÓREA NATIVA: BASE PARA EL DISEÑO DE SISTEMAS AGROFORESTALES EN UNA COMUNIDAD MAYA EN LA PENÍNSULA DE YUCATÁN, MÉXICO.

NATIVE TREE DIVERSITY: BASIS FOR THE DESIGN OF AGROFORESTRY SYSTEMS IN A MAYAN COMMUNITY IN THE YUCATAN PENINSULA, MEXICO.

\section{POLIBETÁNICA}

Instituto Politécnico Nacional
Núm. 51: 73-89 México. Enero 2021

DOI: 10.18387 polibotanica.51.5 


\section{DIVERSIDAD ARBÓREA NATIVA: BASE PARA EL DISEÑO DE SISTEMAS AGROFORESTALES EN UNA COMUNIDAD MAYA EN LA PENÍNSULA DE YUCATÁN, MÉXICO}

\section{NATIVE TREE DIVERSITY: BASIS FOR THE DESIGN OF AGROFORESTRY SYSTEMS IN A MAYAN COMMUNITY IN THE YUCATAN PENINSULA, MEXICO}

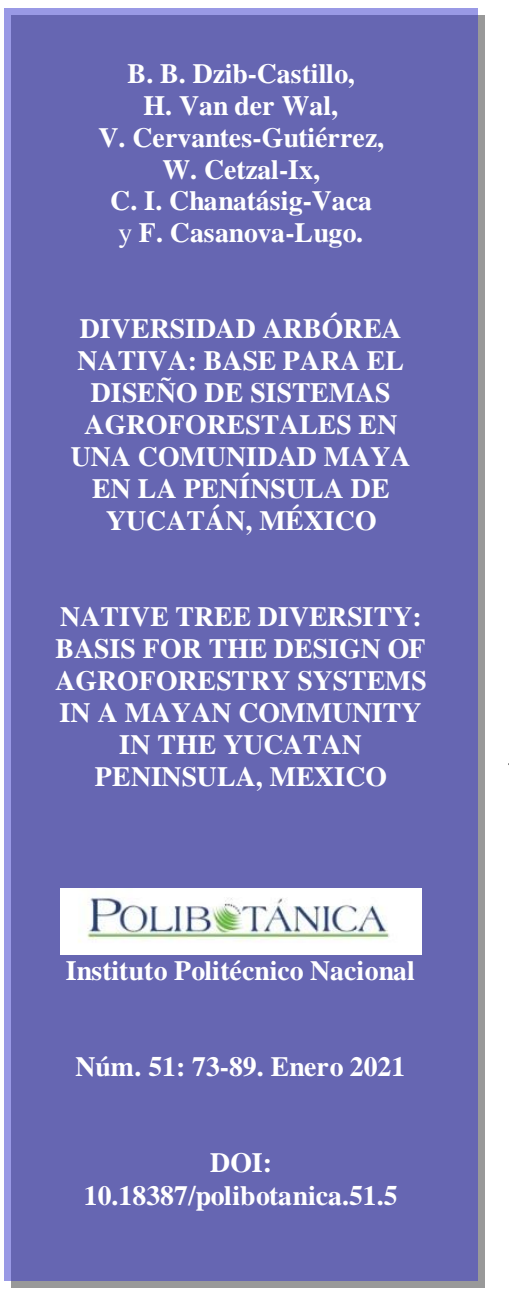

B. B. Dzib-Castillo,

H. Van der Wal,

W. Cetzal-Ix,

C. I. Chanatásig-Vaca

y F. Casanova-Lugo.

DIVERSIDAD ARBÓREA

NATIVA: BASE PARA EL

DISENO DE SISTEMAS

I LA PENINSULA DE

NATIVE TREE DIVERSITY:

BASIS FOR THE DESIGN OF

AGROFORESTRY SYSTEMS

IN A MAYAN COMMUNITY

IN THE YUCATAN

PENINSULA, MEXICO

\section{POLIBETÁNICA}

Instituto Politécnico Nacional

Núm. 51: 73-89. Enero 2021

10.18387/polibotanica.51.5

\author{
B. B. Dzib-Castillo \\ Tecnológico Nacional de México / I.T. Chiná, Campeche, México. \\ H. Van der Wal \\ El Colegio de la Frontera Sur, Unidad Tabasco, Tabasco, México. \\ V. Cervantes-Gutiérrez \\ Universidad Autónoma Metropolitana-Xochimilco, Ciudad de México, México. \\ W. Cetzal-Ix \\ Tecnológico Nacional de México / I.T. Chiná, Campeche, México. \\ C. I. Chanatásig-Vaca \\ Universidad Internacional Iberoamericana \\ Campeche, Campeche, México \\ F. Casanova-Lugo/ fkzanov@gmail.com \\ Tecnológico Nacional de México /I.T. Zona Maya, Quintana Roo, México.
}

RESUMEN: Los sistemas de producción agropecuarios (SPA) en la Península de Yucatán se han analizado con intenciones a su transformación agroforestal. Sin embargo, son escasos los intentos por diseñar sistemas agroforestales desde una perspectiva de integrar a la vegetación natural $(\mathrm{VN})$ con los SPA y de aprovechar el potencial de capacidad de rebrote de la mayoría de las especies nativas. El objetivo del presente estudio fue caracterizar los sistemas de producción agrícola y la vegetación natural, y proponer diseños agroforestales con base en dicha caracterización en la comunidad de Pomuch, Campeche, México. Se entrevistaron a 54 productores y se visitaron los SPA para identificar las actividades realizadas en cada parcela. En áreas con VN se establecieron 24 sitios circulares de $500 \mathrm{~m}^{2}$. Se identificaron los SPA: plantación forestal y frutícola, ganadería, y agricultura (mecanizada, semi-mecanizada y de roza, tumba y quema) y huertos familiares. En áreas con VN se identificaron 45 especies arbóreas, siendo dominantes: Lysiloma latisiliquum, Bursera simaruba y Piscidia piscipula. Se proponen alternativas que incorporan el componente arbóreo, considerando las características de los SPA. Propuestas agroforestales a nivel de parcela buscan favorecer la conectividad entre los SPA y la VN, a través de la creación de conectores de vegetación arbórea que generen un paisaje menos fragmentado e interconectado al incluir árboles en los monocultivos de maíz, dentro y alrededor de los potreros, así como en zonas de transición entre las zonas agropecuarias y la $\mathrm{VN}$, utilizando especies arbóreas nativas de la región como L. latisiliquum, B. simaruba, $P$. piscipula y Platymiscium yucatanum.

Palabras clave: agricultura; agroforestería; conectividad; huerto familiar; paisaje; selvas secundarias; selva baja caducifolia. 
ABSTRACT: The agricultural production systems (APS) in the Yucatan Peninsula (YP) have been analyzed with intentions to their agroforestry transformation. However, the resources for designing agroforestry from a perspective of integration to the natural vegetation (NV) with the APS and to take advantage of the potential of regeneration capacity of most of the native species. The objective is design and characterize the APS and the VN according to the natural resources in the YP, in the community of Pomuch, Campeche, Mexico. Information was collected through interviews with 54 farmers and visits to the APS to identify productive activities. In the areas with NV, 24 temporary circular sites of $500 \mathrm{~m}^{2}$ was established. The APS were identified: forestry and fruit plantation, livestock, and agriculture (mechanized, semimechanized, and slash and burn) and home gardens. In areas with NV, 45 tree species were identified, dominant species were: Lysiloma latisiliquum, Bursera simaruba and Piscidia piscipula. Considering production systems characteristics, alternatives to incorporate trees in the APS were proposed. Agroforestry plot-level proposals, seek to achieve connectivity between the APS and NV, through the creation of connectors for tree vegetation that generate a less fragmented and interconnected landscape by including trees in corn monocultures, in and around the paddocks, as well as in transition zones between agricultural areas and natural vegetation, using tree species native to the region such as $L$. latisiliquum, B. simaruba, $P$. piscipula and Platymiscium yucatanum.

Keywords: agriculture; agroforestry; connectivity; landscape; secondary forests.

\section{INTRODUCCIÓN}

En los trópicos la pérdida neta de bosques entre 2000 y 2005 fue de 9.1 millones de hectáreas de vegetación por años (FAO \& JRC, 2012). Este tema es de particular importancia en México, debido a que la deforestación que se registra en la parte tropical es mucho mayor con respecto a otras partes del país (Isaac-Márquez, 2004). Entre 1978 y 2000 se registró una pérdida total de cubierta forestal en el sureste de México de 4,179,000 ha. En este sentido, Chiapas, Yucatán y Campeche perdieron una cobertura total de 2,909,956 ha, principalmente en bosques caducifolios y subcaducifolios (Díaz-Gallegos et al., 2010). Se ha señalado como factores determinantes del proceso de deforestación a la siembra de cultivos comerciales, la demanda de productos agrícolas para la alimentación y la generación de energía (Lambin \& Meyfroidt, 2011). Aunado a políticas oficiales y programas de subsidio que motivan el establecimiento de grandes extensiones de agricultura mecanizada con monocultivos (Schmink, 1995; Bocco et al., 2001), resultando en la homogenización del paisaje en áreas extensas y la pérdida de biodiversidad (Holzschuh et al., 2007).

La transformación del paisaje ha sido un fenómeno recurrente en la península de Yucatán (PY). En los años 70's y 80's se establecieron monocultivos en los terrenos llamados bajos (selvas bajas inundables), áreas hasta entonces no cultivadas debido a su inundación temporal en el periodo de lluvias; asimismo, se estimuló el abandono de la agricultura de roza, tumba y quema en los lomeríos, lo que propició la regeneración de la vegetación desde ese entonces (PatFernandez et al., 2007). Esta transformación se acentuó en 1995 cuando se puso en marcha el programa Alianza para el Campo, el cual incluyó varios subprogramas para financiar el desarrollo rural a través de equipamiento y mecanización rural para el establecimiento de praderas en las áreas de planicies (Reyes-Hernández et al., 2003).

Para contrarrestar el impacto de los monocultivos en las áreas planas o bajos, se propuso iniciativas como establecimiento de sistemas agroforestales, los cuales permiten a los productores obtener alimentos para sus familias y al mismo tiempo conservan y obtienen diversos bienes y servicios de los recursos forestales (Holzschuh et al., 2007).

A nivel de paisaje, las parcelas cultivadas y las áreas con vegetación natural conforman un entorno diverso de componentes estructurales y funcionales donde cada elemento tiene un espacio y rol establecidos. Estos componentes más que formar una yuxtaposición de simples 
elementos, se interrelacionan y no pueden separarse (Popa, 2012) generando un sistema agroforestal a nivel de paisaje, donde las parcelas agropecuarias interactúan con las áreas remanentes de vegetación.

En la PY se han analizado los sistemas de producción agropecuarios con miras a su transformación agroforestal (Haggar et al., 2001; Snook, 2004), dichos análisis indican que los productores generalmente están dispuestos a experimentar con diseños agroforestales y a incorporar especies maderables con valor comercial en sus parcelas agrícolas. Sin embargo, son escasos los intentos de diseñar agroforestería desde una perspectiva de integración de especies de la vegetación regional con los sistemas agrícolas (Cervantes-Gutierrez et al., 2014). Lo anterior sería posible aprovechando el gran potencial de la capacidad de rebrotar de la mayoría de las especies nativas (Román-Dañobeytia et al., 2014), considerando que las especies que rebrotan tienen un gran potencial de resiliencia y compiten con mayor éxito comparadas con las plántulas de vivero (Macario-Mendoza, 2003). Para aprovechar la actitud de los productores hacia la agroforestería y favorecer la integración de la vegetación regional en estas prácticas, se deberían identificar los métodos más apropiados para la incorporación de especies maderables nativas con valor comercial dentro de las áreas agropecuarias, tomando en consideración las características de cada sistema de producción e identificando las áreas donde los árboles pueden desarrollarse sin afectar la producción de los cultivos agrícolas.

Por lo tanto, la integración de especies arbóreas nativas en los sistemas de producción agrícola permitiría entonces incrementar la conectividad entre las áreas remanentes de vegetación y áreas fragmentadas por la agricultura (Lawrence et al., 2004). Además, representaría hábitats fuera de las áreas naturales protegidas para la conservación de especies (Bhagwat et al., 2008), debido a que promueven la conectividad del paisaje al minimizar el impacto que generan las actividades antropogénicas en el paisaje y sin afectar la producción de los cultivos agrícolas existentes. Por lo anteriormente planteado, el objetivo del presente estudio fue caracterizar los sistemas de producción agrícola y la vegetación natural, y con ello proponer diseños agroforestales con base en dicha caracterización en la comunidad de Pomuch, Campeche, México.

\section{MATERIAL Y MÉTODOS}

\section{Área de estudio}

El estudio se realizó en la temporada de secas (febrero a julio del 2016) en la comunidad de Pomuch, localizada al suroeste de la PY, México. La zona se ubica en una planicie pedregosa del tipo Rendzinas con selva baja caducifolia, que colinda con la zona de manglares, y un área de lomeríos que rodea valles de diferentes extensiones (Zamora-Crescencio, 2003). La precipitación y temperatura media anual son de $1,113 \mathrm{~mm}$ y $26.9^{\circ} \mathrm{C}$. El clima es de tipo $\mathrm{Aw}_{0}$, con lluvias en verano, cinco meses secos y lluvia invernal menor de 5\% (Orellana-Lanza et al., 1999). En esta comunidad existe un total de 8694 pobladores (INEGI, 2010), de los cuales, el $74 \%$ de la población es de origen indígena Maya (CDI, 2010).

\section{Entrevistas y sistemas productivos}

Se seleccionó la comunidad Maya de Pomuch, ubicada en el municipio de Hecelchakán, Campeche, México considerando que esta comunidad conserva tradiciones agrícolas precolombinas como la milpa. Dentro de esta comunidad se entrevistaron al azar a 54 productores, $10 \%$ de los 540 productores activos. Todos fueron hombres de entre 30 y 60 años de edad con estudios básicos (primaria, secundaria). A estos productores se les aplicó un cuestionario con preguntas cerradas para obtener información sobre los sistemas de producción (SP), superficies sembradas, número de parcelas por productor, tipos y métodos de establecimiento de los cultivos, presencia de árboles dentro y alrededor de los diferentes SP, insumos empleados y labores de cultivo. Se visitaron 170 parcelas de los productores entrevistados donde se determinaron sus coordenadas geográficas. Se categorizaron los 
sistemas de producción a partir de los datos generados distinguiendo, a partir de la tecnología de la producción, entre agricultura mecanizada, agricultura semi-mecanizada, agricultura de roza tumba y quema (sistema de producción manual, el cual cada determinado tiempo cambia de sitio, para lo cual se derriba vegetación natural para establecerse, siendo un método de cultivo rotativo que se establece en zonas con lomeríos), agricultura de espeque (sistema de producción manual que se práctica en un solo lugar, sin tener que derribar vegetación natural para establecerse cada determinado tiempo), ganadería, fruticultura, plantación forestal, y huerto familiar. Posteriormente, los datos obtenidos fueron analizados con el programa Excel $2016 \circledR$, con el cual se identificó y clasificó la información para responder a los objetivos planteados.

\section{Caracterización de la vegetación natural}

Se realizó un muestreo en las áreas con vegetación natural (VN) remanente, tanto en lomeríos como en planicies, alrededor de las áreas de uso agropecuario para identificar la estructura y composición arbórea, estableciendo 24 sitios temporales, los cuales se eligieron de forma aleatoria. Para esto se utilizaron parcelas circulares de $500 \mathrm{~m}^{2}$, donde se midieron todos los árboles mayores a $10 \mathrm{~cm}$ de DAP (diámetro a la altura del pecho) a $1.3 \mathrm{~m}$ de altura desde el suelo, así como la altura total. En el área central de la parcela se estableció una subparcela de $100 \mathrm{~m}^{2}$ donde se tomaron los mismos datos para cada árbol medido entre 3 y $10 \mathrm{~cm}$ de DAP. Se identificaron las especies en campo por su nombre local y se recolectaron muestras botánicas para la identificación de las especies en el herbario Universiada Autónoma de Campeche y con el listado de Carnevali et al. (2010). Con el programa ILWIS 3.31® (Koolhoven et al., 2007), se desplegaron las coordenadas de las parcelas sobre una imagen base del área del 2016 del Google Earth®, ubicando la distribución de las parcelas cultivadas.

\section{RESULTADOS}

\section{Caracterización de los sistemas de producción}

La superficie de los sistemas de producción (SP) en promedio fue de $2.5 \pm 1.92$ hectáreas, presentándo variaciones dentro de cada uno en su tamaño y número de SP (Tabla 1). El número promedio de parcelas por productor fue de 3.08 \pm 1.36 ; en cuanto a plantaciones forestales solamente se encontró una plantación de cedro dentro de los sistemas de producción agrícola (SPA), tuvieron mayor presencia el sistema semi-mecanizado (SMM) y el sistema mecanizado (SM). En ambos SPA fue común la presencia de policultivos, pero la frecuencia fue mayor en el SMM (94\% de parcelas de policultivo). En el caso de la agricultura de espeque, $78 \%$ de las parcelas fueron de policultivo, mientras fue menor en parcelas de SM (58\%). La densidad de siembra del maíz fue 25\% mayor en el SM (63 900 plantas ha $^{-1}$ ) con respecto al SMM (48 560 plantas $\mathrm{ha}^{-1}$ ), tanto en policultivo como en monocultivo (Tabla 1).

El cultivo de maíz (Zea mays L.) en Pomuch se realiza con semilla criolla y comercial. En el SM, 9\% de los productores utilizan semilla criolla y $91 \%$ semilla comercial; y en el SMM, $31 \%$ utiliza semilla criolla y $69 \%$ semilla comercial. El maíz es el cultivo predominante en la producción agrícola, aunque en su mayoría los productores (47) combinan el maíz con calabaza menuda (Cucurbita moschata Duchesne) y en menor medida la chihua (Cucurbita mixta Pangalo) (Tabla 2).

\section{Sistemas de producción y especies arbóreas}

Los 54 productores entrevistados poseen un huerto familiar, y consideran que este aporta diversos productos a la economía familiar (Tabla 1). En estos huertos se encontró seis especies de árboles maderables provenientes de la VN, mantenidas por los productores para diversos usos (Tabla 3). En el caso del cultivo de árboles frutales, el $18 \%$ del total de los productores cuentan con parcelas frutícolas con una superficie promedio de 1,33 $\pm 0,87$ ha, las cuales cultivan de tres formas: dispersos dentro de las áreas agrícolas, en forma de monocultivos o en 
plantaciones mixtas (árboles de diferentes especies) en áreas compactas; sin embargo, muchas de estas plantaciones ya son viejas y requieren ser renovadas.

De las 170 parcelas visitadas, $4.7 \%$ estuvieron dedicadas a la producción ganadera (de tipo extensiva y abocada a la producción de bovinos) (Tabla 1). La presencia de árboles en los potreros es visible, en su totalidad por 13 especies maderables nativas, cuatro de estas utilizadas como cercas vivas y las restantes dispersas al interior de los potreros como sombra o para alimentación de los animales (Tabla 4).

Tabla 1. Descripción de los sistemas de producción identificados en el ejido de Pomuch, Campeche, México. NP = Número de parcelas. PAS = Promedio de área por sistema (ha). FU = Frecuencia de uso: A: Anual $(+=5$ a 6 años y descansos por un mismo lapso; $++=$ siembra en junio y cosecha en diciembre y enero; +++ siembra en junio y cosecha en enero-diciembre). P: Permanente. TSE = Topografía del sitio de establecimiento. DSCP = Densidad de siembra del cultivo principal. (*) Área urbana. Actividades de manejo: Manejo mínimo (MM), Siembra con espeque

(SCE), deshierbe manual (DM), aplicación de herbicidas (AH), aplicación de fertilizantes (AF)

\begin{tabular}{|c|c|c|c|c|c|c|c|}
\hline $\begin{array}{l}\text { Sistema de } \\
\text { producción }\end{array}$ & NP & PAS (ha) & FU & TSE & Productos & DSCP & Actividades de manejo \\
\hline $\begin{array}{l}\text { Huerto } \\
\text { familiar }\end{array}$ & 54 & $0,17 \pm 0,24$ & $\mathrm{P}$ & Plana* & $\begin{array}{l}\text { Especies frutales, } \\
\text { maderables, } \\
\text { medicinales y } \\
\text { ornamentales } \\
\end{array}$ & N/A & MM \\
\hline $\begin{array}{l}\text { Agricultura } \\
\text { de Rosa, } \\
\text { Tumba y } \\
\text { Quema }\end{array}$ & 1 & 0,50 & $\mathrm{~A}(+)$ & Ladera & $\begin{array}{c}\text { Maíz (cultivo principal, } \\
\text { rendimiento promedio } \\
\text { de } 1 \text { ton } \text { ha }^{1} \text { ) } \\
\text { intercalado con } \\
\text { calabaza menuda } \\
\end{array}$ & $\begin{array}{l}30,000 \\
\text { plantas } \\
\text { ha }^{-1}\end{array}$ & SE, DM, AH \\
\hline $\begin{array}{l}\text { Agricultura } \\
\text { de Espeque }\end{array}$ & 15 & $1,53 \pm 1,49$ & $\begin{array}{c}\text { A } \\
(++)\end{array}$ & $\begin{array}{c}\text { Plana y } \\
\text { pedregosa }\end{array}$ & $\begin{array}{c}\text { Maíz (cultivo } \\
\text { principal) intercalado } \\
\text { con calabaza menuda y } \\
\text { chihua. }\end{array}$ & $\begin{array}{l}41,850 \\
\text { plantas } \\
\text { ha }^{-1}\end{array}$ & SE, DM, AH, AF \\
\hline $\begin{array}{l}\text { Agricultura } \\
\text { Semi- } \\
\text { mecanizada }\end{array}$ & 39 & $2,02 \pm 1,02$ & $\begin{array}{c}\text { A } \\
(++)\end{array}$ & Plana & $\begin{array}{l}\text { Maíz intercalado con } \\
\text { calabaza menuda y } \\
\text { chihua }\end{array}$ & $\begin{array}{c}48,560 \\
\text { plantas } \\
\text { ha }^{-1}\end{array}$ & $\begin{array}{l}\text { SE, DM, AH (preparación } \\
\text { del terreno con maquinaria, } \\
\text { cosecha de forma manual) }\end{array}$ \\
\hline $\begin{array}{l}\text { Agricultura } \\
\text { Mecanizada }\end{array}$ & 34 & $3,68 \pm 2,36$ & $\begin{array}{c}\text { A } \\
(+++)\end{array}$ & Plana & $\begin{array}{c}\text { Maíz (cultivo } \\
\text { principal) se combina } \\
\text { con otros cultivos tal } \\
\text { como en los otros } \\
\text { sistemas } \\
\end{array}$ & $\begin{array}{c}63,900 \\
\text { plantas } \\
\text { ha }^{-1}\end{array}$ & $\begin{array}{l}\text { Preparación del terreno y } \\
\text { siembra con maquinaria, } \\
\text { algunas actividades de } \\
\text { forma manual. Uso de AH } \\
\text { y AF }\end{array}$ \\
\hline Fruticultura & 18 & $1,33 \pm 0,87$ & $\mathrm{P}$ & Plana & $\begin{array}{c}\text { Mango (Manguifera } \\
\text { indica), naranja dulce } \\
\text { (Citrus sinensis), } \\
\text { ciruelo (Spondias } \\
\text { purpurea) }\end{array}$ & Variable & $\begin{array}{l}\text { MM (plantaciones viejas y } \\
\text { con pocos cuidados) }\end{array}$ \\
\hline Ganadería & 8 & $8,19 \pm 5,87$ & $\mathrm{P}$ & $\begin{array}{l}\text { Plana y } \\
\text { con } \\
\text { laderas }\end{array}$ & $\begin{array}{c}\text { Ganadería (tipo } \\
\text { extensivo), enfocada a } \\
\text { bovinos para producir } \\
\text { becerras que amplíen o } \\
\text { mantengan el hato, } 10 \\
\text { animales en promedio } \\
\text { por productor }\end{array}$ & N/A & $\begin{array}{l}\text { MM (potreros con pastos } \\
\text { naturales, se chapean para } \\
\text { favorecer su crecimiento, se } \\
\text { mantienen con cercas vivas } \\
\text { y postes de madera muerta } \\
\text { y con reemplazo cada cinco } \\
\text { años) }\end{array}$ \\
\hline Forestal & 1 & 0,12 & $\mathrm{P}$ & Plana & $\begin{array}{c}\text { Especie maderable }(C . \\
\text { odorata) con manejo } \\
\text { mínimo }\end{array}$ & $\begin{array}{c}100 \\
\text { árboles } \\
\text { (aprox.) }\end{array}$ & MM \\
\hline
\end{tabular}


Tabla 2. Superficie total plantada para los principales cultivos anuales en Pomuch, Campeche, considerando una muestra de 54 productores. NP = Número de productores. STS $=$ Superficie total sembrada. SPC = Superficie por cultivo.

\begin{tabular}{lcccc}
\hline Nombre científico & Nombre común & NP & STS (ha) & SPC $(\%)$ \\
\hline Zea mays & Maíz & 53 & 215.1 & 52.98 \\
Cucurbita moschata & Calabaza menuda & 35 & 115.6 & 28.47 \\
Cucurbita mixta & Calabaza chihua & 24 & 41.2 & 10.15 \\
Phaseolus lunatus & Ibes & 13 & 20.9 & 5.15 \\
Phaseolus vulgaris & Fríjol de guía & 12 & 6.2 & 1.53 \\
Vigna unguiculata & Xpelon & 4 & 5.8 & 1.43 \\
Ipomoea batatas & Camote & 2 & 0.5 & 0.12 \\
Arachis hypogaea & Cacahuate & 1 & 0.4 & 0.1 \\
Citrullus lanatus & Sandía & 1 & 0.2 & 0.05 \\
Pachyrhizus erosus & Jícama & 1 & 0.1 & 0.02 \\
\hline
\end{tabular}

Tabla 3. Especies maderables presentes en áreas de vegetación natural y en huertos familiares del ejido de Pomuch, Campeche, México.

\begin{tabular}{lcc}
\hline \multicolumn{1}{c}{ Especie } & $\begin{array}{c}\text { Huerto familiar } \\
\text { (individuos ha }^{-1} \text { ) }\end{array}$ & $\begin{array}{c}\text { Vegetación natural } \\
\text { (individuos ha }^{-1} \text { ) }\end{array}$ \\
\hline Lysiloma latisiliquum (L.) Benth & 1 & 593 \\
Bursera simaruba (L.) Sarg. & 1 & 323 \\
Piscidia piscipula (L.) Sarg. & 3 & 174 \\
Havardia albicans Britton \& Rose & 0.1 & 23 \\
Caesalpinia violacea (Mill.) Standl. & 1 & 8 \\
Apoplanesia paniculata C. Presl & 2 & 3 \\
\hline
\end{tabular}

Tabla 4. Especies presentes dentro de los sistemas de producción ganadero del ejido de Pomuch, Campeche, México. Abreviaturas: presente (+), No presente (-).

\begin{tabular}{lccc}
\hline Especies & $\begin{array}{c}\text { Especies al interior del } \\
\text { potrero }\end{array}$ & $\begin{array}{c}\text { Especies vivas en } \\
\text { cercas }\end{array}$ & $\begin{array}{c}\text { Especies muertas en } \\
\text { cercas }\end{array}$ \\
\hline Lysiloma latisiliquum (L.) Benth & + & - & - \\
Bursera simaruba (L.) Sarg. & + & + & - \\
Piscidia piscipula (L.) Sarg. & + & + & + \\
Gymnopodium floribundum Rolfe & + & - & - \\
Mimosa bahamensis Benth. & + & - & + \\
Thouinia paucidentata Radlk. & + & - & - \\
Vitex gaumeri Greenm. & + & + & + \\
Caesalpinia gaumeri Greenm. & + & + & + \\
Havardia albicans Britton \& Rose & + & - & + \\
Acacia gaumeri S.F. Blake & + & - & - \\
Caesalpinia violacea (Mill.) Standl. & + & - & - \\
Spondias purpurea L. & + & - & - \\
Tecoma stans Juss. & + & & + \\
\hline
\end{tabular}




\section{Distribución geográfica de los sistemas de producción}

Los huertos familiares se registraron en la zona urbana del ejido, las parcelas ganaderas y frutícolas en la cercanía de la comunidad, a una distancia menor a $6 \mathrm{~km}$. Las parcelas frutícolas se identificaron en áreas compactas cerca de los pozos en los valles; las parcelas agrícolas en los valles desde menos de uno hasta $20 \mathrm{~km}$ de distancia al área urbana de la comunidad. Algunas parcelas recién desmontadas están en los valles pequeños que se encuentran dispersos entre los lomeríos, al suroeste de la población (fig. 1). La agricultura mecanizada con monocultivos se encuentra a más de $10 \mathrm{~km}$ del pueblo, los policultivos y agricultura semimecanizada están distribuidos a diferentes distancias, desde el pueblo hasta los límites del ejido. Las parcelas de agricultura de espeque se dividen en parcelas cercanas en las tierras pedregosas y planas al oeste del poblado, y parcelas más alejadas en áreas recién desmontadas que serán destinadas a la agricultura semi-mecanizada en el futuro. Esto muestra que existe una fragmentación del paisaje que era dominado por una matriz forestal y que actualmente está siendo dominada por los sistemas agropecuarios. El establecimiento de corredores con árboles a través de los sistemas agropecuarios permitiría disminuir la fragmentación uniendo la vegetación natural.

\section{Vegetación natural}

El área de muestreo fue en total 1.2 ha (24 sitios de $500 \mathrm{~m}^{2}$ ), de esta superficie 0.4 ha ( 8 sitios) correspondieron al área de planicie y 0.8 ha al área de lomeríos (16 sitios). Se identificó un total de 45 especies arbóreas, 20 de estas se encontraron en ambas condiciones topográficas, 16 en planicie y nueve en lomeríos (Tabla 5). Con respecto a las categorías diamétricas, 20 especies presentaron individuos en categorías de DAP $>10 \mathrm{~cm} \mathrm{y} \geq 3 \leq 10 \mathrm{~cm}, 22$ no presentaron individuos con DAP $>10 \mathrm{~cm}$, y tres sólo presentaron individuos con DAP $>10 \mathrm{~cm}$ (Tabla 5).

En cuanto al área basal estimada, para la zona de planicies y lomeríos se obtuvieron $9 \mathrm{~m}^{2} \mathrm{ha}^{-1} \mathrm{y}$ $11.6 \mathrm{~m}^{2} \mathrm{ha}^{-1}$, respectivamente. En ambos casos la mayor área basal fue aportada por los árboles con DAP > $10 \mathrm{~cm}, 7.7 \mathrm{~m}^{2} \mathrm{ha}^{-1}$ y $9.5 \mathrm{~m}^{2} \mathrm{ha}^{-1}$, respectivamente; por su parte, árboles con DAP $\leq 10 \mathrm{~cm}$ tuvieron menor área basal, $1.3 \mathrm{~m}^{2} \mathrm{ha}^{-1}$ en planicies y $2.1 \mathrm{~m}^{2} \mathrm{ha}^{-1}$ para lomeríos. En planicies y lomeríos las especies con mayor área basal fueron Lysiloma latisiliquum (L.) Benth., Bursera simaruba (L.) Sarg. y Piscidia piscipula (L.) Sarg., las cuales en conjunto aportaron $87 \%$ y $75 \%$, respectivamente (Tabla 5). En áreas planas, las especies L. latisiliquum, B. simaruba, P. piscipula, Plumeria obtusa L. y Vitex gaumeri Greenm. con DAP $>10 \mathrm{~cm}$, aportaron $97 \%$ del total del área basal; mientras en la categoría diamétrica $<10 \mathrm{~cm}, 97 \%$ del área basal se distribuyó entre 17 especies. Por su parte, en el área de lomeríos, L. latisiliquum, B. simaruba, P. piscipula, V. gaumeri y Diospyros cuneata con DAP $>10 \mathrm{~cm}$, aportaron $94 \%$ del área basal, a diferencia de la categoría diamétrica entre 3 y $10 \mathrm{~cm}$, donde el 93\% del área basal se distribuyó entre 16 especies (Tabla 5).

Se estimaron 940 individuos por hectárea en el área plana y 1404 en el área de lomeríos. Dentro de la categoría diamétrica $\leq 10 \mathrm{~cm}$ se estimó un total de 1419 individuos ha ${ }^{-1}$, mientras que en la categoría $>10 \mathrm{~cm}$ de DAP se estimaron 925 individuos ha $^{-1}$. La densidad de individuos por hectárea fue mayor en la categoría entre 3 y $10 \mathrm{~cm}$ de DAP tanto en lomeríos como en planicies (Tabla 5). Considerando las dos categorías diamétricas y las dos condiciones topográficas, se identificaron seis especies que comparten ambas condiciones topográficas: Harvadia albicans, L. latisiliquum, B. simaruba, Caesalpinia gaumeri Greenm., P. piscipula y Vitex gaumeri. De todas las especies, las de mayor número de individuos por hectárea fueron: L. latisiliquum, B. simaruba, Lonchocarpus xuul Lundell, P. piscipula y G. floribundum Rolfe (Tabla 5).

De las especies con mayor número de individuos, en cada categoría diamétrica por condición topográfica, L. latisiliquum presentó el mayor número de individuos por hectárea en la categoría diamétrica mayor a $10 \mathrm{~cm}$ en ambas condiciones topográficas, mientras que en la categoría entre 3 y $10 \mathrm{~cm}$ de DAP en lomeríos, $L$. xuul fue la especie con el mayor número de individuos por hectárea y G. floribundum para el área de planicies (Tabla 5). 

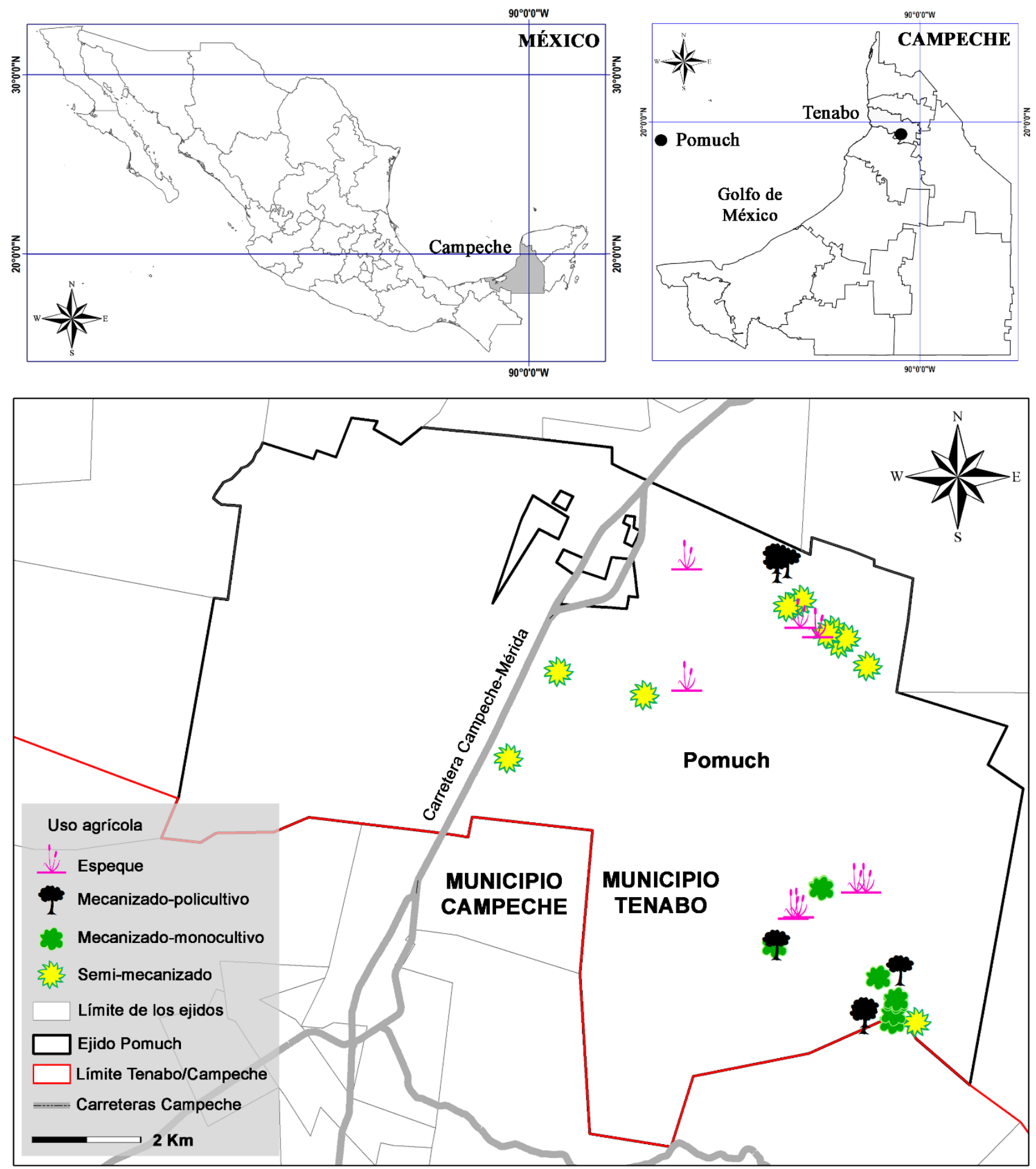

Fig. 1. Distribución de sistemas de producción en Pomuch, Campeche, México. 
Tabla 5. Número de individuos y porcentaje de área basal (AB) por especie en selva baja caducifolia del ejido de Pomuch, Hecelchakán, Campeche, México. $\mathrm{DAP}=$ diámetro a la altura del pecho.

\begin{tabular}{|c|c|c|c|c|c|c|c|c|}
\hline \multirow{3}{*}{ Especies } & \multicolumn{4}{|c|}{ Loma } & \multicolumn{4}{|c|}{ Plano } \\
\hline & \multicolumn{2}{|c|}{ DAP $>10 \mathrm{~cm}$} & \multicolumn{2}{|c|}{ DAP 3-10 cm } & \multicolumn{2}{|c|}{ DAP $>10 \mathrm{~cm}$} & \multicolumn{2}{|c|}{ DAP 3-10 cm } \\
\hline & Individuos ha ${ }^{-1}$ & $\mathrm{AB}(\%)$ & Individuos ha ${ }^{-1}$ & $\mathrm{AB}(\%)$ & Individuos ha ${ }^{-1}$ & $\mathrm{AB}(\%)$ & Individuos ha ${ }^{-1}$ & $\mathrm{AB}(\%)$ \\
\hline Acacia gaumeri S.F. Blake & 3 & 0.4 & 4 & 0.6 & & & 3 & 0.6 \\
\hline Acacia milleriana Standl. & 1 & 0.1 & & & & & 5 & 0.9 \\
\hline Apoplanesia paniculata C. Presl & 3 & 0.2 & & & & & & \\
\hline Astronium graveolens Jacq. & & & & & & & 8 & 0.6 \\
\hline Bauhinia jenningsii P.Wilson & & & 5 & 0.7 & & & & \\
\hline Bourreria pulchra Millsp. ex Greenm. & 1 & 0.1 & 30 & 3.2 & & & 8 & 0.9 \\
\hline Bunchosia glandulosa DC. & & & 5 & 0.8 & & & 3 & 1.2 \\
\hline Bursera simaruba (L.) Sarg. & 146 & 26.7 & 56 & 12.5 & 88 & 23.9 & 33 & 6.2 \\
\hline Caesalpinia gaumeri Greenm & 3 & 0.5 & 5 & 0.5 & 3 & 0.3 & 30 & 3.7 \\
\hline Caesalpinia vesicaria $\mathrm{L}$. & & & 4 & 0.3 & & & & \\
\hline Caesalpinia violacea (Mill.) Standl. & & & & & 8 & 1.2 & & \\
\hline Cnidoscolus multilobus (Pax.) I.M. Johnston & & & & & & & 3 & 0.1 \\
\hline Coccoloba barbadensis Jack. & 1 & 0.2 & 11 & 1.6 & & & & \\
\hline Cochlospermum vitifolium (Willd.) Spreng. & 3 & 0.3 & & & & & 3 & 0.4 \\
\hline Croton reflexifolius Kunth & 4 & 0.4 & 9 & 0.6 & & & & \\
\hline Dendropanax arboreus (L.) Decne. \& Planch. & & & 3 & 0.3 & & & & \\
\hline Diospyros cuneata Standl. & 24 & 3.0 & 61 & 8.5 & & & 5 & 1.3 \\
\hline Erythroxylum confusum Britton & & & 4 & 0.4 & & & & \\
\hline Eugenia yucatanensis Standley & & & 10 & 0.7 & & & 13 & 1.1 \\
\hline Exostema caribaeum (Jacq.) Roem. \& Schult. & & & 19 & 1.5 & & & 20 & 2.1 \\
\hline Gliricidia sepium ( Jacq. ) Kunth & 1 & 0.1 & 5 & 0.5 & & & & \\
\hline Guettarda elliptica $\mathrm{Sw}$. & & & 35 & 2.1 & & & 30 & 2.6 \\
\hline
\end{tabular}




\begin{tabular}{|c|c|c|c|c|c|c|c|c|}
\hline \multirow{3}{*}{ Especies } & \multicolumn{4}{|c|}{ Loma } & \multicolumn{4}{|c|}{ Plano } \\
\hline & \multicolumn{2}{|c|}{ DAP> $10 \mathrm{~cm}$} & \multicolumn{2}{|c|}{ DAP 3-10 cm } & \multicolumn{2}{|c|}{ DAP> $>10 \mathrm{~cm}$} & \multicolumn{2}{|c|}{ DAP 3-10 cm } \\
\hline & Individuos ha ${ }^{-1}$ & AB (\%) & Individuos ha ${ }^{-1}$ & AB (\%) & Individuos ha ${ }^{-1}$ & $\mathrm{AB}(\%)$ & Individuos ha ${ }^{-1}$ & AB (\%) \\
\hline Gymnopodium floribundum Rolfe & 5 & 0.5 & 41 & 5.5 & & & 83 & 10.2 \\
\hline Hampea trilobata Standl. & & & 4 & 0.4 & & & & \\
\hline Havardia albicans Britton \& Rose & 5 & 1.1 & 3 & 0.6 & 5 & 1.4 & 10 & 3.5 \\
\hline Hintonia octomera (Hemsley) Bullock & & & & & & & 3 & 0.2 \\
\hline Hyperbaena winzerlingii Sandl. & & & 1 & 0.2 & & & & \\
\hline Jatropha gaumeri Greenm. & & & & & & & 10 & 1.4 \\
\hline Lonchocarpus xuul Lundell & 13 & 1.5 & 228 & 21.1 & & & & \\
\hline Lysiloma latisiliquum (L.) Benth & 223 & 48.4 & 45 & 9.4 & 220 & 62.8 & 105 & 34.4 \\
\hline Mimosa bahamensis Benth & 4 & 0.4 & 38 & 3.7 & & & 53 & 5.3 \\
\hline Neomillspaughia emarginata S.F.Blake & & & 5 & 0.3 & & & 8 & 0.5 \\
\hline Piscidia piscipula (L.) Sarg. & 71 & 10.4 & 35 & 7.8 & 35 & 7.0 & 33 & 8.9 \\
\hline Platymiscium yucatanum Stand. & 4 & 0.4 & 11 & 2.4 & & & & \\
\hline Plumeria obtusa L. & & & & & 8 & 1.7 & 3 & 1.1 \\
\hline Psidium sartorianum (O.Berg) Nied. & & & & & & & 3 & 0.3 \\
\hline Randia aculeata $\mathrm{L}$. & & & 33 & 1.7 & & & 58 & 4.2 \\
\hline Senna atomaria (L.) H.S.Irwin \& Barneby & & & & & & & 3 & 0.2 \\
\hline Sickingia salvadorensis Standl & & & 1 & 0.2 & & & & \\
\hline Sideroxylon americanum (Miller) T.D.Penn. & 1 & 0.1 & 83 & 5.8 & & & & \\
\hline Spondias purpurea $\mathrm{L}$. & & & 3 & 0.2 & & & & \\
\hline Swartzia cubensis (Britton \& P. Wilson) Standl. & 1 & 0.1 & & & & & & \\
\hline Tecoma stans Juss & & & & & & & 3 & 0.6 \\
\hline Thouinia paucidentata Radlk & & & 48 & 3.9 & & & 25 & 4.7 \\
\hline Vitex gaumeri Greenm. & 35 & 5.0 & 13 & 2.1 & 10 & 1.8 & 10 & 2.5 \\
\hline TOTAL & 550 & 100 & 854 & 100 & 375 & 100 & 565 & 100 \\
\hline
\end{tabular}




\section{DISCUSIÓN}

Al caracterizar los SP en el ejido Pomuch, se observó la ausencia de árboles integrados dentro de los SPA; sin embargo, el componente arbóreo (CA) sí está presente en los sistemas ganaderos, en las plantaciones de cultivos frutales y en la única plantación forestal presente en el ejido. Para integrar el CA dentro de los SP es necesario considerar la densidad de siembra del cultivo principal (García et al., 2001). En el caso del SP mecanizado, donde la densidad de siembra del maíz es la más alta, la integración de árboles tendría que realizarse en los límites de las parcelas.

Otro aspecto importante es la combinación de diferentes cultivos anuales en una misma superficie, ya que los productores lo practican comúnmente. Aunque no se observó la presencia del CA dentro de este SP, podrían integrarse, debido a la baja densidad del cultivo principal (maíz). Esto no afectaría de forma inmediata la producción de maíz y aumentaría la probabilidad de obtener productos de especies con distintos requerimientos ecológicos en el mismo espacio. Según García et al. (2001) y Limongi-Andrade et al. (2004), las ventajas de combinar árboles con maíz son la madera y forraje, reducción de la erosión y aumento de la fertilidad del suelo, y aumento de la capacidad de almacenamiento de agua en el suelo, así como la conservación de especies vegetales y animales.

En los sistemas de producción ganadero se identificaron árboles maderables nativos dispersos al interior de los potreros (Tabla 4), su presencia indica la posibilidad de introducirlos en estos sistemas; dichas especies podrían aportar forraje y mejorar el suelo, transformándolos en sistemas silvopastoriles más productivos (Villanueva-Partida et al., 2019). En los monocultivos con árboles frutales, se observaron árboles viejos que requieren ser renovados, abriendo la posibilidad de integrar cultivos agrícolas de ciclo corto mientras los árboles aún son pequeños, cuando la sombra de las copas cierre el dosel se podrían integrar cultivos que soporten sombra; esto permitiría incrementar la eficiencia del uso de la tierra y el ingreso económico para el productor (Gao et al., 2013).

Respecto al ámbito forestal, el que exista una sola plantación con una especie maderable dentro de la comunidad, sugiere que los productores no identifican a este tipo de plantaciones como un medio para obtener recursos económicos; posiblemente la $\mathrm{VN}$ cubre las necesidades de madera de la población, mientras las plantaciones forestales para aserrío tienen un retorno económico a mediano y largo plazo. Una manera de incentivar el establecimiento de árboles, sería establecerlos en diferentes áreas de las parcelas agropecuarias, utilizándolos como hospederos o precursores de especies que ofrezcan un beneficio a corto y largo plazo. Un ejemplo sería el cultivo de pitahaya (Hylocereus undatus (Haw.) Britton \& Rose) que crece sobre los árboles, aporta frutos anualmente y genera recursos económicos a través de su comercialización (Cálix de Dios et al., 2014; Castillo-Martínez et al., 2014).

En Pomuch existen áreas de vegetación forestal remanente en lomeríos y planos, éstas son relictos de lo que antes fue un área forestal más amplia y que actualmente se encuentra fragmentada por el uso agrícola, principalmente en las áreas planas. En estas áreas existen recursos maderables utilizados por los productores para cubrir sus necesidades de madera para leña y construcción. Estos recursos no tienen un manejo planificado y son aprovechados indiscriminadamente, afectando las poblaciones de las especies maderables más demandadas (p. ej. Cedrela odorata L. y Platymiscium yucatanum Standl.), las cuales deben considerarse para realizar trabajos de enriquecimiento dentro de las áreas con $\mathrm{VN}$, estableciendo plantaciones en brechas o claros dentro de estas áreas. Además, que algunas de esas especies pueden ser integradas a los sistemas productivos generando sistemas agroforestales que mantengan la diversidad y aporten recursos maderables a los productores (García et al., 2001; LimongiAndrade et al., 2004).

Dentro de esta VN se presenta un proceso de regeneración que está sustentado por el mayor número de individuos entre 3 y $10 \mathrm{~cm}$ de DAP en la mayoría de las especies, lo que puede 
atribuirse al abandono de la agricultura de roza-tumba y quema en los lomeríos en los años 80 's y debido a que el mayor porcentaje de área basal bajo ambas condiciones topográficas, solo se registró en L. latisiliquum (demandada comercialmente). Es evidente que en los fragmentos de VN, los productores podrían propiciar la presencia de especies con mayor valor comercial, promoviendo su mejor desarrollo a través de aclareos (Lamb et al., 2005).

La presencia de altos porcentajes de área basal de una especie con demanda en el mercado, permitiría su aprovechamiento mediante prácticas de extracción de bajo impacto, tales como aprovechar diámetros mayores, reforestar o incentivar la regeneración de dichas especies, certificación de aprovechamientos que disminuyan los efectos sobre el suelo y agua (Sist, 2000; Orozco-Vílchez, 2004). Con la información obtenida en la VN, se plantean algunas combinaciones de cultivos agrícolas con especies maderables nativas, las cuales buscan propiciar la interconexión, aumentar la biodiversidad y disminuir la fragmentación del paisaje en el ejido de Pomuch.

\section{Árboles al interior de los cultivos de maíz}

Maíz-Jabín en callejones. El "jabín” (Piscidia piscipula) es una leguminosa que fija nitrógeno atmosférico, restituyendo el extraído por los cultivos y/o pastos dándole sostenibilidad al SP. Es una especie que tolera la poda, generando rebrotes aún con podas severas, lo cual permitiría podarla cuando el exceso de sombra generado por su copa afecte el cultivo agrícola aledaño y usar su follaje en la alimentación del ganado o como materia orgánica en el suelo. Su madera es de buena calidad para la construcción de viviendas, preferida como leña y para la elaboración de carbón, además las flores permiten obtener miel de buena calidad (Zamora-Crescencio et al., 2009); además, es una de las especies más abundantes en las áreas de VN del ejido de Pomuch (Tabla 5). Estas características la hacen una especie con alto potencial para ser aprovechada por los productores, estableciéndola en hileras dentro de los SPA de espeque y semi-mecanizado, aprovechando la baja densidad del cultivo de maíz. La densidad de los árboles dentro del cultivo debe ser baja para limitar la sombra de los árboles sobre el cultivo agrícola y permitir el uso de maquinaria agrícola (fig. 2).

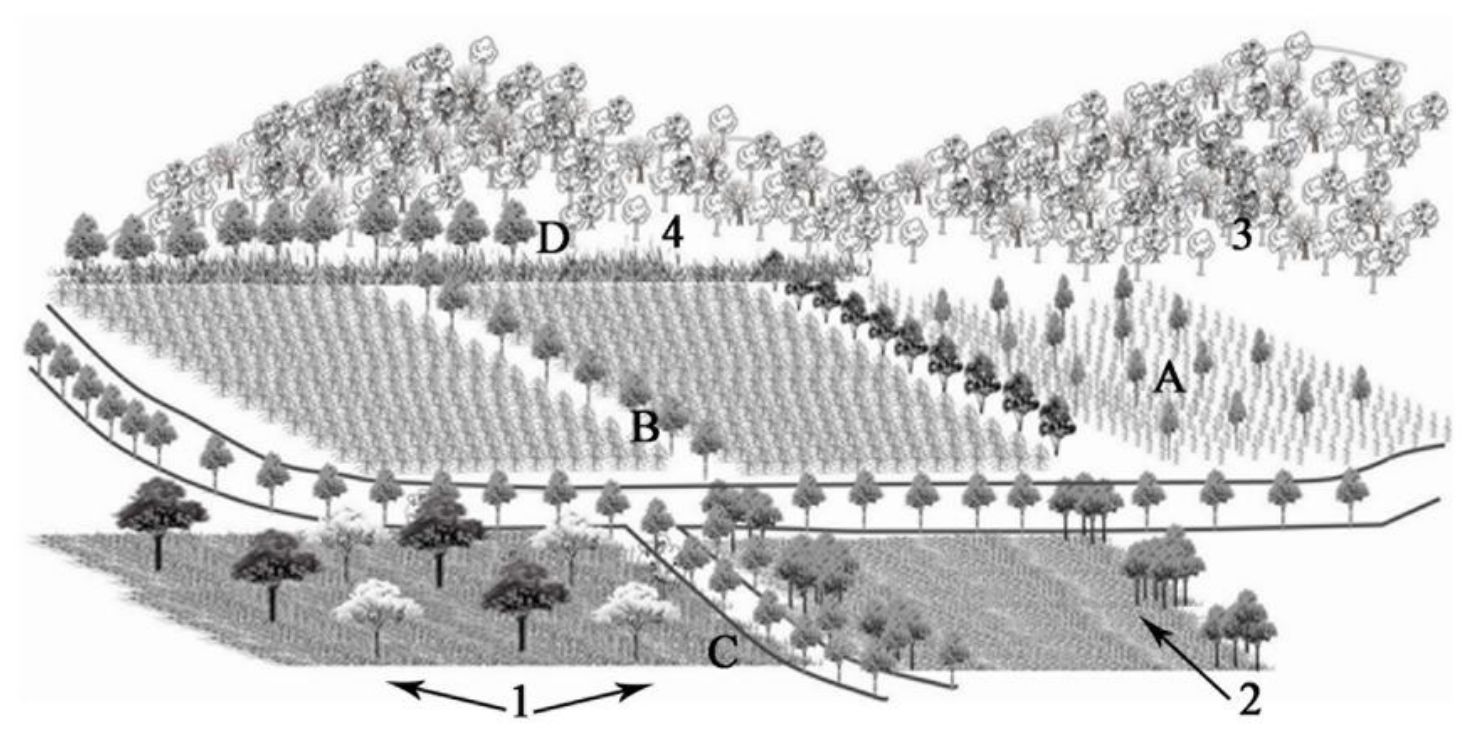

Fig. 2. Opciones para la introducción del componente arbóreo dentro de un entorno productivo en el ejido de Pomuch, Campeche, México. A. Árboles de P. piscipula intercalados con cultivos de Z. mays. B. árboles de especies maderables nativas ( $C$. odorata y $P$. yucatanum) en bordes de caminos y límites de parcelas. C. distribución de árboles nativos al interior de los potreros en forma dispersa (1) y agrupados (2). D. establecimiento de árboles en áreas de transición entre áreas de cultivos y áreas con VN en lomeríos. (3) antes de la introducción de árboles y (4) después de la introducción de árboles. 


\section{Árboles en bordes}

Una forma de conectar fragmentos forestales es por medio del establecimiento de árboles en línea entre los límites de las parcelas y caminos (fig. 2). En Pomuch una alternativa sería el uso del granadillo ( $P$. yucatanum) y el cedro $(C$. odorata), ambas son especies nativas con demanda en el mercado por su madera de alta calidad. Lo anterior motivaría propagarlas y establecerlas, ya que los resultados del muestreo indican poca presencia del granadillo y nula presencia de cedro en las áreas de VN (Tabla 5). Por otro lado, existe la opción de utilizar a B. simaruba, considerando que es un árbol que enraíza fácilmente y se puede obtener árboles a partir de ramas o postes establecidos en los cercos, además de puede funcionar como tutor de otras especies de plantas, como la pitahaya (Hylocereus spp.) (Cálix de Dios et al., 2014), entre otras funciones como planta forrajera y medicinal. El establecerlos en los límites de parcelas y caminos mejoraría el paisaje, disminuiría la tala de árboles para cercas con postes, y se dispersarían las semillas hacia áreas cercanas.

\section{Árboles en potreros}

Los árboles dentro de los sistemas de producción ganadero pueden aportar forraje con alto valor proteico, madera, leña, frutas, fijación de nitrógeno atmosférico al suelo y sombra para el ganado (Zamora-Crescencio et al., 2009; Casanova-Lugo et al., 2014). Para este sistema se propone incrementar la densidad de $P$. piscipula y L. latisiliquum, ambas presentes en los potreros (Tabla 4) y en áreas de VN, también representadas en ambas categorías diamétricas y condiciones topográficas (Tabla 5). Estas especies son importantes por su valor económico de la madera, pueden fijar nitrógeno al suelo, además que tienen la característica de generar brotes nueves después de una poda convirtiéndose en una opción para la obtención de forraje para el ganado.

Otras especies nativas que deberían integrarse son el ramón (Brosimum alicastrum Sw.) y el pixoy (Guazuma ulmifolia Lam.), a pesar de que no se encontraron en los potreros y en la VN, su importancia radica en que son especies que aportan forraje para la alimentación del ganado (Sosa-Rubio et al., 2004), ya que toleran la poda de sus ramas y pueden rebrotar sin problema (Casanova-Lugo et al., 2014). El establecimiento de árboles al interior de los potreros puede ser de forma dispersa, aunque otra opción es integrarlos en grupos en áreas determinadas para disminuir el efecto de la sombra sobre el pasto (Córdoba-Sánchez \& Hernández-Daumás, 2003; Villanueva-Partida et al., 2016), o alrededor de cuerpos de agua que sirven de abrevadero para los animales (fig. 2).

Franjas de árboles en la zona de transición entre áreas cultivadas (áreas planas) y áreas con vegetación secundaria (áreas de lomeríos)

El uso inadecuado del fuego por parte de los productores para eliminar restos de cultivos anteriores y vegetación no deseada en áreas de agricultura, ha propiciado la perdida de $\mathrm{VN}$ en la zona de transición entre planos y lomeríos (fig. 2); favoreciendo la colonización de pastos y plantas anuales. Una opción para contrarrestar la presencia de especies no deseadas en las zonas de transición, es la incorporación de especies arbóreas maderables nativas de uso local y con valor comercial, evitaría que los productores se preocupen por quemar estas áreas. Dos especies que podrían utilizarse son $P$. piscipula y Platymiscium yucatanum, ambas apreciadas por su madera, y considerando que la siembra directa de sus semillas ha probado ser factible (Tabla 5) (Dzib-Castillo et al., 2012).

\section{CONCLUSIONES}

Se observó la ausencia de árboles dentro los sistemas de producción agrícola, sin embargo, existe la mezcla de cultivos agrícolas, lo cual muestra un conocimiento sobre la combinación de diferentes especies en una misma área cultivada. El huerto familiar fue el sistema agroforestal prevaleciente, en donde la presencia de árboles maderables nativos sugiere que los productores si consideran mantener o plantar árboles. La ganadería representa una opción agroforestal ya 
que, si mantiene árboles tanto al interior como en los límites de las pasturas, con opción a ser mejorado. El cultivo de maíz se realiza a baja densidad, principalmente los que utilizan el método de siembra manual (espeque), esta baja densidad ofrece la posibilidad de establecer árboles en límites entre parcelas, caminos, y dentro del cultivo, generando sistemas agroforestales a nivel de parcela y paisaje.

Existe un proceso de sucesión en la vegetación remanente, donde existe una mayor presencia de árboles jóvenes, tanto en lomeríos como en planos; de las 45 especies identificadas, $L$. latisiliquum presentó el mayor valor del área basal por hectárea, esto podría aprovecharse para establecer un plan de manejo y aprovechar el recurso maderable de forma sostenible, considerando el valor comercial de esta especie. Otras especies como B. simaruba, P. piscipula que se encontraron en grandes cantidades dentro de la vegetación natural son candidatas para formar parte preponderante de los sistemas agroforestales, además de incluir especies que son nativas y que han desaparecido prácticamente de la vegetación natural como P. yucatanum y $C$. ododrata, las cuales se pueden recuperar mediante la inclusión en sistemas agroforestales como en enriquecimientos dentro de la vegetación natural.

Las opciones agroforestales diseñadas integran las especies nativas en los diferentes sistemas de producción identificados, tomando en cuenta los recursos con que se cuentan en esta región, y que se requieren una evaluación a través del tiempo, para determinar la factibilidad socioeconómica y ecológica de los mismos.

\section{AGRADECIMIENTOS}

A todos los productores del ejido de Pomuch que colaboraron en la realización de este trabajo. También agradecemos al Tecnológico Nacional de México / IT de Chiná por todas las facilidades brindadas.

\section{LITERATURA CITADA}

Bhagwat, S. A., Willis, K. J., Birks, H. J. B., \& Whittaker, R. J. (2008). Agroforestry: a refuge for tropical biodiversity? Trends in Ecology and Evolution, 23(5), 261-267. https://doi.org/10.1016/j.tree.2008.01.005

Bocco, G., Mendoza, M., \& Masera, O. R. (2001). La dinámica del cambio del uso del suelo en Michoacán.Una propuesta metodológica para el estudio de los procesos de deforestación. Investigaciones Geograficas, 44, 18-38. https://doi.org/10.14350/rig.59133

Cálix de Dios, H., Castillo-Martínez, R., \& Caamal-Canché, H. J. (2014). Caracterización de la producción de pitahaya (Hylocereus spp.) en la zona maya de Quintana Roo. Agroecología, 9(1-2), 123-132.

Carnevali, F. C. G., Tapia-Muñoz, J. L., Duno de Stefano, R., \& Ramírez-Morillo, I. (2010). Flora Ilustrada de la Península de Yucatán: Listado Florístico (Primera ed). Centro de Investigación Científica de Yucatán, A. C.

Casanova-Lugo, F., Petit-Aldana, J., Solorio-Sánchez, F. J., Parsons, D., \& Ramírez-Avilés, L. (2014). Forage yield and quality of Leucaena leucocephala and Guazuma ulmifolia in mixed and pure fodder banks systems in Yucatan, Mexico. Agroforestry Systems, 88(1), 29-39. https://doi.org/10.1007/s10457-013-9652-7

Castillo-Martínez, R., Ebel, R., Cálix de Dios, H., Ferral Piña, J., Nava Padilla, R., \& Cach Chuc, F. (2014). Paquete técnico para la producción orgánica de pitahaya (Primera ed). Universidad de Quintana Roo.

CDI. (2010). Indicadores económicos sobre la población indígena desagregada por municipio. Comisión Nacional Para El Desarrollo de Los Pueblos Indígenas. 
https://datos.gob.mx/busca/dataset/poblacion-indigena-por-municipio

Cervantes-Gutierrez, V., Gama-Castro, J. E., Roldan-Aragón, I. E., \& Hernández-Cárdenas, G. (2014). Basis for Implementing Restoration Strategies: San Nicolás Zoyatlan SocialEcological System (Guerrero, Mexico). Terra Latinoamericana, 32(2), 143-159.

Córdoba-Sánchez, E., \& Hernández-Daumás, S. (2003). Competencia por luz en sistemas silvopastoriles. Ecofronteras, 18, 21-23.

Díaz-Gallegos, J. R., Mas, J. F., \& Velázquez, A. (2010). Trends of tropical deforestation in Southeast Mexico. Singapore Journal of Tropical Geography, 31(2), 180-196. https://doi.org/10.1111/j.1467-9493.2010.00396.x

Dzib-Castillo, B. B., Van Der Wal, H., Chanatásig-Vaca, C. I., Macario-Mendoza, P. A., \& PatFernández, J. M. (2012). Emergencia de plántulas de especies maderables nativas de la Península de Yucatán. Revista Mexicana de Ciencias Forestales, Vol 3, no. http://www.scielo.org.mx/pdf/remcf/v3n10/v3n10a7.pdf

FAO, \& JRC. (2012). Global forest land-use change 1990-2005. In E. J. Lindquist, R. D'Annunzio, A. Gerrand, M. Kenneth, F. Achard, R. Beuchle, A. Brink, H. D. Eva, P. Mayaux, J. San-Miguel-Ayanz, \& H.-J. Stibig. (Eds.), FAO (FAO Forest). Food and Agriculture Organization of the United Nations and European Commission Joint Research Centre. http://www.fao.org/docrep/017/i3110e/i3110e.pdf

Gao, L., Xu, H., Bi, H., Xi, W., Bao, B., Wang, X., Bi, C., \& Chang, Y. (2013). Intercropping competition between apple trees and crops in agroforestry systems on the loess plateau of China. PLoS ONE, 8(7), 1-8. https://doi.org/10.1371/journal.pone.0070739

García, E., Jaime, M., Mejía, B., Guillén, L., \& Harvey, C. A. (2001). Árboles dispersos dentro de cultivos anuales en el municipio de Ilobasco, El Salvador. Agroforestería En Las Américas, 8(31), 39-44.

Haggar, J., Ayala, A., Díaz, B., \& Reyes, C. U. (2001). Participatory design of agroforestry systems: Developing farmer participatory research methods in Mexico. Development in Practice, 11(4), 417-424. https://doi.org/10.1080/09614520120066701

Holzschuh, A., Steffan-Dewenter, I., Kleijn, D., \& Tscharntke, T. (2007). Diversity of flowervisiting bees in cereal fields: Effects of farming system, landscape composition and regional context. Journal of Applied Ecology, 44(1), 41-49. https://doi.org/10.1111/j.1365-2664.2006.01259.x

INEGI. (2010). Censo de población y vivienda 2010. Instituto Nacional de Estadística y Geografía. https://www.inegi.org.mx/programas/ccpv/2010/

Isaac-Márquez, R. (2004). Explorando la perspectiva campesina de la agroforestería en la reserva de la biósfera de Calakmul. Universidad y Ciencia, 20, 39-54.

Koolhoven, W., Hendrikse, J., Nieuwenhuis, W., Retsios, B., Schouwenburg, M., Wang, L., Budde, P., \& Nijmeijer, R. (2007). ILWIS 3.31. Overijssel: Universidad de Twente. http://ilwis.itc.utwente.nl/wiki/index.php/Main_Page

Lamb, D., Erskine, P. D., \& Parrotta, J. A. (2005). Restoration of degraded tropical forest landscapes. Science, 310(5754), 1628-1632. https://doi.org/10.1126/science.1111773

Lambin, E. F., \& Meyfroidt, P. (2011). Global land use change, economic globalization, and the looming land scarcity. Proceedings of the National Academy of Sciences of the United States of America, 108(9), 3465-3472. https://doi.org/10.1073/pnas.1100480108

Lawrence, G., Cheshire, L., \& Richards, C. A. (2004). Agricultural production and the ecological question. In R. White (Ed.), Controversies in Environmental Sociology (pp. 221-237). Cambridge University Press. https://doi.org/10.1017/CBO9780511804434.014

Limongi-Andrade, R., Harvey, C. A., Jiménez, F., \& Tamara, B. (2004). Caracterización del sistema agroforestal maíz con árboles dispersos en la cuenca del Rio Carrizal, Manabí, Ecuador. Agroforestería En Las Américas, 41-42, 50-55.

Macario-Mendoza, P. A. (2003). Efecto del cambio de uso del suelo sobre la selva y estrategias para el manejo sustentable de la vegetación secundaria en Quintana Roo. Universidad Autónoma de Yucatán.

Orellana-Lanza, R., Balam-Kú, M., Bañuelos, I. R., García, E., González-Iturbide, A. J. A., Herrera, C. F., \& Vidal, L. J. (1999). Evaluación climática. In P. A. Chico Ponce de 
Recibido:

8/julio/2020

Aceptado:

9/febrero/2021
León (Ed.), Atlas de procesos territoriales de Yucatán (Primera ed, pp. 163-182). Universidad Autónoma de Yucatán.

Orozco-Vílchez, L. (2004). Planificación del manejo diversificado de bosques latifoliados húmedos tropicales. In Catie (Issue 56). http://www.fao.org/fileadmin/user_upload/training_material/docs/Planificación_del manejo diversificado de bosques.pdf

Pat-Fernandez, A. L., Nahed-Toral, J., Parra-Vázquez, M. R., Nazar-Beutelspacher, D. A., García-Barrios, L., Bello-Baltazar, E., \& Herrera-Hernández, O. B. (2007). Modos de vida y seguridad alimentaria de los mayas de Campeche. In REDSAN-FAO (Ed.), Memoria. Concurso Red SAN 2007 (Primera ed, pp. 128-167). Organización de las Naciones Unidas para la Agricultura y Alimentación.

Popa, A. (2012). Aspects of Agricultural Landscape As a Cultural Asset in Metropolitan Areas: Case Study for Bucharest City. Mokslas - Lietuvos Ateitis, 4(2), 128-134. https://doi.org/10.3846/mla.2012.25

Reyes-Hernández, H., Cortina-Villar, S., Perales-Rivera, H., Kauffer-Michel, E., \& PatFernández, J. M. (2003). Efecto de los subsidios agropecuarios y apoyos gubernamentales sobre la deforestación durante el período 1990-2000 en la región de Calakmul, Campeche, México. Investigaciones Geograficas, 51, 88-106. https://doi.org/10.14350/rig.30416

Román-Dañobeytia, F. J., Levy-Tacher, S. I., Macario-Mendoza, P., \& Zúñiga-Morales, J. (2014). Redefining secondary forests in the mexican forest code: Implications for management, restoration, and conservation. Forests, 5(5), 978-991. https://doi.org/10.3390/f5050978

Schmink, M. (1995). La matriz socioeconómica de la deforestación. In M. F. Paz (Ed.), De bosques y gente: aspectos sociales de la deforestación en América Latina. (Primera ed, pp. 17-51). Universidad Nacional Autónoma de México-Centro Regional de Investigaciones Multidisciplinarias.

Sist, P. (2000). Reduced-impact logging in the tropics : objectives, principles and impacts. International Forestry Review, 2(1), 3-10.

Snook, A. (2004). En busca de sistemas agroforestales viables: resultados y experiencias en Calakmul y la zona maya. In N. Armijo \& C. Llorens (Eds.), Uso, conservación y cambio en los bosques de Quintana Roo Chetumal: Universidad de Quintana Roo (Primera ed, pp. 131-158). Universidad de Quintana Roo.

Sosa-Rubio, E. E., Pérez-Rodríguez, D., Ortega-Reyes, L., \& Zapata-Buenfil, G. (2004). Evaluación del potencial forrajero de árboles y arbustos tropicales para la alimentación de ovinos. Técnica Pecuaria En México, 42(2), 129-144.

Villanueva-Partida, C., Casanova-Lugo, F., Villanueva-López, G., González-Valdivia, N., Oros-Ortega, I., \& Díaz-Echeverría, V. (2016). Influence of the density of scattered trees in pastures on the structure and species composition of tree and grass cover in southern Tabasco, Mexico. Agriculture, Ecosystems and Environment, 232, 1-8. https://doi.org/10.1016/j.agee.2016.07.020

Villanueva-Partida, C. R., Casanova-Lugo, F., González-Valdivia, N. A., Villanueva-López, G., Oros-Ortega, I., Cetzal-Ix, W., \& Basu, S. K. (2019). Traditional uses of dispersed trees in the pastures of the mountainous region of Tabasco, Mexico. Agroforestry Systems, 93(2), 383-394. https://doi.org/10.1007/s10457-017-0125-2

Zamora-Crescencio, P. (2003). Contribución al estudio florístico y descripción de la vegetación del municipio de Tenabo, Campeche, México. Polibotánica, 15, 1-40.

Zamora-Crescencio, P., Flores-Guido, J. S., \& Ruenes-Morales, R. (2009). Flora útil y su manejo en el cono sur del estado de Yucatán, México. Polibotánica, 28, 227-250. 